\title{
CD147 Induces Epithelial-to-Mesenchymal Transition by Disassembling Cellular Apoptosis Susceptibility Protein/E-Cadherin/ $\beta$-Catenin Complex in Human Endometriosis
}

Chaoqun Wang, ${ }^{*}$ Jieting Zhang, ${ }^{*}$ Kin L. Fok, ${ }^{*}$ Lai L. Tsang, ${ }^{*}$ Mei Ye, ${ }^{\dagger}$ Jianni Liu, ${ }^{\dagger}$ Fanghong Li, ${ }^{\dagger}$ Allan Z. Zhao, ${ }^{\dagger}$ Hsiao C. Chan, ${ }^{*}$ and Hao Chen ${ }^{* \dagger}$

From the Epithelial Cell Biology Research Center,* School of Biomedical Sciences, Faculty of Medicine, The Chinese University of Hong Kong, Shatin, Hong Kong; the Institute of Biomedical and Pharmaceutical Sciences, ${ }^{\dagger}$ Guangdong University of Technology, People's Republic of China; and the Sichuan University-The Chinese University of Hong Kong Joint Laboratory for Reproductive Medicine, ${ }^{\ddagger}$ West China Second University Hospital, Sichuan University, Sichuan, People's Republic of China

Accepted for publication

March 19, 2018.

Address correspondence to Hao Chen, Ph.D., Institute of Biomedical and Pharmaceutical Sciences, Guangdong University of Technology, No. 100, Waihuan Xi Road, Guangzhou Higher Education Mega Center, People's Republic of China, 510006; or Hsiao Chang Chan, Ph.D., Epithelial Cell Biology Research Center, School of Biomedical Sciences, Faculty of Medicine, The Chinese University of Hong Kong, Shatin, Hong Kong. E-mail: chenhao@gdut.edu. cn or chenhaojob@yahoo.com, or hsiaocchan@cuhk.edu.hk.

\begin{abstract}
Epithelial-to-mesenchymal transition (EMT) is postulated to be a prerequisite for the establishment of endometriosis (EMS), a common reproductive disorder in women. Our previous studies have demonstrated the elevated expression of transmembrane glycoprotein CD147 and its prosurvival effect on abnormal cells in endometriosis. Intriguingly, CD147 is known to promote EMT in cancers. However, the involvement of CD147 in EMT during the establishment of endometriosis remains incompletely understood. We found that CD147 promotes EMT in human endometrial adenocarcinoma cell line Ishikawa. We identified a novel CD147-interacting partner, cellular apoptosis susceptibility protein (CAS), which stabilized the interaction between E-cadherin (E-cad) and $\beta$-catenin ( $\beta$-cat) by forming the CAS/E-cad/ $\beta$-cat complex. Down-regulation of CAS led to the release and nuclear translocation of $\beta$-cat from E-cad, resulting in the overexpression of the EMT-promoting gene SNAIL. Interestingly, overexpression of CD147 impaired the interaction between CAS and E-cad and triggered the release of $\beta$-cat from the CAS/E-cad/ $\beta$-cat complex, which in turn led to EMT. Furthermore, CAS was downregulated in EMS, with elevated levels of CD147 and nuclear $\beta$-cat. These findings suggest a previously undefined role of CAS in regulating EMT and reveal the involvement of a CD147-induced EMT signaling pathway in pathogenic progression of EMS. (Am J Pathol 2018, 188: 1597-1607; https:// doi.org/10.1016/j.ajpath.2018.03.004)
\end{abstract}

Endometriosis (EMS) affects $6 \%$ to $10 \%$ of women of reproductive age with pelvic pain and infertility. ${ }^{1-3}$ It originates from retrograde menstruation of endometrial cells, which migrate through the fallopian tubes and invade into the peritoneal cavity to establish ectopic lesions. ${ }^{1,4}$ Although EMS is considered a benign disease, several studies suggest that the enhanced cell migration and invasion during EMS progression is similar to that observed in cancer metastasis. ${ }^{5,6}$

Epithelial-to-mesenchymal transition (EMT) is a critical step in cancer metastasis. It is a process in which polarized epithelial cells are converted into mesenchymal-like cells with stronger motility and invasiveness. $\beta$-catenin ( $\beta$-cat) signaling pathway and cadherin-mediated cell adhesion play important roles in regulating EMT. ${ }^{7}$ The interplay between these two pathways regulates the activity of $\beta$-cat. In cell adhesion, the interaction between cadherin and $\beta$-cat

Supported in part by National Nature Science Foundation of China grants 81671432 (H.C.) and 81370709 (H.C.C.) and Science and Technology Planning Project of Guangdong Province grant 2016A020218005 (H.C.). C.W. was supported by the Hong Kong PhD Fellowship Scheme issued by Research Grants Council of Hong Kong.

Disclosures: None declared.

Current address of C.W., University of California, San Francisco, San Francisco, CA. 
maintains cell polarity. Loss of cadherin-mediated adhesion promotes the nuclear translocation of $\beta$-cat that in turn activates set of EMT-promoting transcription factors, including Snail, Slug, and Twist. These transcription factors subsequently lead to down-regulation of epithelial marker E-cadherin (E-cad) and up-regulation of mesenchymal markers [eg, vimentin and $\mathrm{N}$-cadherin $(\mathrm{N}$-cad)], which are considered the hallmarks of EMT. ${ }^{7-11}$ Because ectopic lesions in EMS demonstrated biological properties similar to those of cancer metastasis in terms of cell survival and cell migration, EMT has been postulated to be involved in the establishment of EMS. In fact, studies have demonstrated the loss of E-cad expression in ectopic lesions of EMS. ${ }^{6}$ However, the molecular mechanisms underlying the regulation of EMT in EMS remain largely unknown.

CD147 is a transmembrane glycoprotein mainly expressed in the reproductive tracts, eye, brain, and muscles in healthy individuals. ${ }^{12}$ Despite the restricted expression in healthy individuals, its expression is widely elevated in different types of cancer cells. CD147 is known to act as a prosurvival and promigration factor in various physiologic and pathologic processes, such as spermatogenesis and tumor metastasis. ${ }^{13-18}$ The promigration property of CD147 can be attributed to its ability in promoting the production of matrix metalloproteinases (MMPs) and in mediating EMT. ${ }^{15,19-21}$ However, the molecular mechanism underlying the regulation of EMT by CD147 remains elusive.

Our previous studies have found that CD147 is elevated in human EMS. ${ }^{17}$ Elevated level of CD147 promotes the abnormal cell survival through up-regulation of Bcl-2. ${ }^{18} \mathrm{On}$ the other hand, it promotes the migration of endometrial cells in an MMP2-independent manner. ${ }^{17}$ However, the potential involvement of CD147-mediated EMT in the establishment of EMS has not been explored. In the present study, we identified a CD147-interacting partner cellular apoptosis susceptibility protein (CAS) and found that CD147 and E-cad formed a complex through CAS. Furthermore, an elevated level of CD147 impaired the binding between CAS and E-cad, leading to the release of $\beta$-cat that subsequently promotes EMT. In addition, the expression of CAS was down-regulated in EMS lesions with elevated levels of CD147 and nuclear $\beta$-cat. Together, our results suggest that the interaction between CD147 and the CAS/E-cad/ $\beta$-cat complex is an important regulator of EMT during progression of EMS.

\section{Materials and Methods}

\section{Human Tissue Collection}

In total, 59 women undergoing gynecologic laparoscopic surgery were recruited to the study: 47 with ovarian EMS (aged 25 to 42 years) and 12 without EMS (aged 26 to 45 years). Endometriotic tissues were collected from the walls of endometriomas in patients with EMS, and normal endometrial tissues (confirmed by pathologic diagnosis) were collected by curettage in women with infertility. All tissues were taken at the proliferative phase of the menstrual cycle, and all women with or without EMS had regular menstrual cycles. Individuals receiving hormone therapy or anti-inflammatory agents were excluded. All samples were collected with informed consent from each woman and approval by the institutional review board of the Second People's Hospital of Shenzhen. ${ }^{17}$

\section{Cell Culture, Plasmid and siRNA Transfection, Lentivirus Transduction, and Anti-CD147 Treatment}

Ishikawa (ISK) cells were cultured in RPMI 1640 medium with $10 \%$ heat-inactivated fetal bovine serum (FBS) and $1 \%$ penicillin-streptomycin in 5\% carbon dioxide incubators at $37^{\circ} \mathrm{C}$. For overexpression of CD147, ISK cells were seeded in six-well plate at $5 \times 10^{5}$ cells per well. The pcDNA3.0CD147 plasmid was provided by Dr. Zhinan Chen (The Fourth Military Medical University, Xi' an, People's Republic of China). ${ }^{22}$ Transfection was performed using $8 \mu \mathrm{L}$ of Lipofectamine 2000 (Invitrogen, Carlsbad, CA) and $5 \mu \mathrm{g}$ of DNA of pcDNA3.0-CD147 or pcDNA3.0 according to the manufacturer's instructions. The transfected cells were selected with $400 \mu \mathrm{g} / \mathrm{mL}$ of G418 for 10 days. For knockdown CAS in ISK cells, the cells were transfected with $50 \mathrm{nmol} / \mathrm{L}$ CAS siRNA as above. The three CAS siRNAs were purchased from Invitrogen (catalog number 1299001). The protein was extracted with radioimmunoprecipitation assay buffer 5 days after transfection. The lentiviral CD147 shRNA and CAS overexpression lentiviruses were purchased from GenePharma (Shanghai, People's Republic of China). For lentiviral transduction, ISK cells were seeded into 24-well plate at $1 \times 10^{4}$ cells per well with $200 \mu \mathrm{L}$ of Dulbecco's modified Eagle's medium and RPMI 1640 plus 10\% FBS. After 1 day in culture, the medium was replaced with fresh medium and cells were transduced with $5 \times 10^{6}$ transducing units of lentiviruses plus $0.1 \%$ polybrene. Stable transduced cells were selected by adding $4 \mu \mathrm{g} / \mathrm{mL}$ of puromycin after 48 hours of transfection. For anti-CD147 antibody treatment, cells were seeded in six-well plate at $5 \times 10^{4}$ cells per well. After 24 -hour culture, the medium was replaced by fresh medium that contained $10 \mu \mathrm{g} / \mathrm{mL}$ of mouse anti-CD147 monoclonal antibody (Table 1), and normal mouse IgG at the same concentration was used as the negative control. After 48-hour antibody treatment, total cell protein was extracted with radioimmunoprecipitation assay buffer.

\section{RNA Extraction and Real-Time Quantitative PCR}

Total RNA from cultured cells and human specimens was extracted using Trizol regent (Invitrogen), according to the manufacturer's instructions. cDNA was synthesized from $1 \mu \mathrm{g}$ of total RNA with iScript cDNA synthesis kit (Bio-Rad Laboratories, Hercules, CA), as per the manufacturer's instructions. The real-time quantitative PCR for SNAIL was performed using the Power SYBR Green PCR Master Mix (Applied Biosystems, Foster City, CA) in a $10-\mu \mathrm{L}$ total volume on Applied Biosystems 7500 Fast Real-Time PCR System, as described 
Table 1 Antibodies Used in This Study

\begin{tabular}{|c|c|c|c|c|}
\hline Name & Experiment & Vendor & Catalog number & Dilution \\
\hline Anti-CD147 (M6/1) & Functional blocking & Santa Cruz Biotechnology, Dallas, TX & sc-51591 & $10 \mu \mathrm{g} / \mathrm{mL}$ \\
\hline Anti-CD147 (N19) & WB/IP & Santa Cruz Biotechnology & sc-9752 & $1: 500(\mathrm{WB}) ; 5 \mu \mathrm{g}(\mathrm{IP})$ \\
\hline Anti-E-cadherin & WB/IP & Santa Cruz Biotechnology & sc-7870 & $1: 500(\mathrm{WB}) ; 5 \mu \mathrm{g}(\mathrm{IP})$ \\
\hline Anti-N-cadherin & WB & Invitrogen, Carlsbad, CA & 333900 & $1: 1000$ \\
\hline Anti-vimentin & WB & Santa Cruz Biotechnology & sc-5565 & $1: 500$ \\
\hline Anti-CAS & WB/IP & Santa Cruz Biotechnology & sc-1708 & $1: 500$ (WB); $5 \mu \mathrm{g}(\mathrm{IP})$ \\
\hline Anti-histone $\mathrm{H} 3$ & WB & Millipore, Burlington, MA & $06-755$ & $1: 2000$ \\
\hline Anti-GAPDH & WB & Santa Cruz Biotechnology & sc- 47724 & $1: 2000$ \\
\hline Anti- $\beta$-actin & WB & Sigma-Aldrich & A1978 & 1:2000 \\
\hline
\end{tabular}

GAPDH, glyceraldehyde-3-phosphate dehydrogenase; IP, immunoprecipitation; WB, Western blot.

previously. ${ }^{18}$ The sequences of the primers are listed as follows: SNAIL (forward: 5'-TTTACCTTCCAGCAGCCCTA-3'; reverse: 5'-CCCACTGTCCTCATCTGACA-3'; product size, $207 \mathrm{bp}$ ) and $\mathrm{GAPDH}$ (forward: 5'-AACGACCCCTTCATTGAC-3'; reverse: 5'-TCCACGACATACTCAGCAC-3'; product size, $190 \mathrm{bp}$ ). The real-time quantitative PCR for $C A S$ expression was performed using TaqMan primers (Applied Biosystems). TaqMan GenExpression Inventoried Assay (assay number Hs00354853_m1 hCAS) and TaqMan Universial PCR Master Mix (Applied Biosystems) were used in real-time PCR reaction. S18 rRNA (Applied Biosystems) was used as the endogenous control. The PCR programs were as follows: stage $1,50^{\circ} \mathrm{C}$ for 2 minutes; stage $2,95^{\circ} \mathrm{C}$ for $10 \mathrm{mi}-$ nutes; and stage $3,95^{\circ} \mathrm{C}$ for 15 seconds and $60^{\circ} \mathrm{C}$ for 1 minute. Stage 3 was repeated for 40 cycles. The PCR assays were performed in separate tubes, and relative quantitation of mRNA was determined using standard curve method according to the manufacturer's instructions.

\section{Cell Migration and Invasion Assay}

Cell migration assay was performed by live imaging system (Carl Zeiss, Oberkochen, Germany). ${ }^{23}$ Briefly, $1 \times 10^{6}$ cells were cultured in a well of the six-well plate until they reached a confluent monolayer. This monolayer was subsequently scratched with a $20-\mu \mathrm{L}$ culture tip, and the medium was replaced with fresh medium plus $1 \%$ FBS. Cellular migration from the two wound fronts was tracked and recorded during a 24-hour period using a live imaging system. At least five random imaging views were investigated on each plate to quantify the distance of migration. For invasion assay, the Corning BioCoat Matrigel Invasion Chambers with an 8.0- $\mu \mathrm{m}$ PET Membrane (Corning Inc., Corning, NY) were used, according to the manufacturer's instructions. Briefly, $1 \times 10^{5}$ cells were resuspended in RPMI 1640 medium without FBS and plated into the upper inserts. RPMI 1640 medium that contained 20\% FBS was added to the lower chamber as a chemoattractant. After 24 hours of incubation, noninvasive cells were removed from the upper chamber with a cotton swab. The cells on the lower surface of the insert were fixed by $4 \%$ paraformaldehyde and stained with $0.5 \%$ crystal violet. The invading cells were counted under microscopy.

\section{Co-Immunoprecipitation, Silver Staining, and Mass Spectrometry}

Co-immunoprecipitation was conducted according to the manufacturer's instructions (GE Healthcare, Little Chalfont, UK). The appropriate antibody $(5 \mu \mathrm{g})$ was added in binding buffer, and $20 \mu \mathrm{L}$ of magnetic bead slurry medium was equilibrated using $500 \mu \mathrm{L}$ of binding buffer. After equilibration, the antibody solution was added in binding buffer with magnetic bead and incubated for 30 minutes. After antibody binding, the beads were washed using $500 \mu \mathrm{L}$ of binding buffer; then the protein sample (lyzed with the immunoprecipitation lysis buffer) was added to the antibody-bond beads and incubated overnight at $4^{\circ} \mathrm{C}$. Then the beads were washed three times with wash buffer. The proteins bound to the beads were eluted by $20 \mu \mathrm{L}$ of $1 \times$ SDS loading buffer. For silver staining, after running the SDS-PAGE gel for the immunoprecipitation protein products, the gel was fixed with fixation solution I (50\% methanol and $10 \%$ acetic acid) for 20 minutes and then fixed with fixation solution II (5\% methanol and 1\% acetic acid) for 10 minutes. The gel was washed three times with double distilled water for 5 minutes. The gel was sensitized with $0.02 \%$ thiosulfate solution for 90 seconds and then washed three times with double distilled water for 30 seconds. After sensitization, gel was stained with 12 $\mathrm{mmol} / \mathrm{L}$ silver nitrate for 30 minutes and then washed three times with double distilled water for 1 minute. The gel was developed in $0.04 \%$ formaldehyde with $2 \%$ sodium carbonate for $10 \mathrm{mi}-$ nutes. Each of the specific protein bands was cut from the gel and analyzed by matrix-assisted laser desorption/ionization time-offlight tandem mass spectrometry. The mass spectrometry analysis was conducted in the Center for Genomic Sciences at the University of Hong Kong. The antibodies used in coimmunoprecipitation assay are listed in Table 1.

\section{Western Blot Analysis}

Cells and human tissues were lyzed with radioimmunoprecipitation assay buffer. Western blot analysis 
A

VC
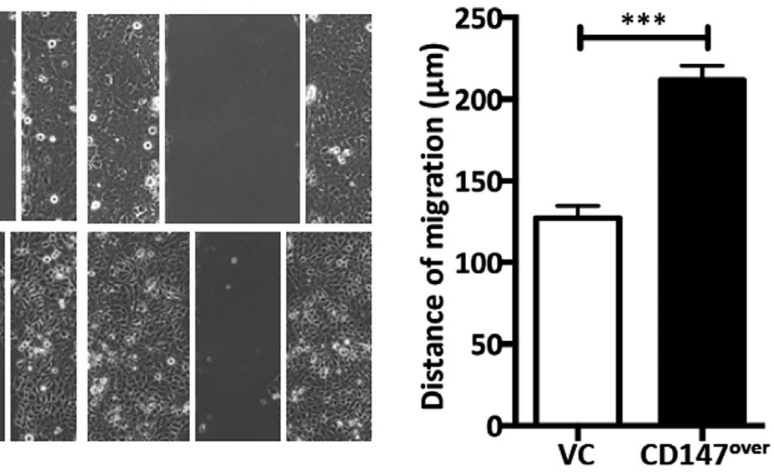

B
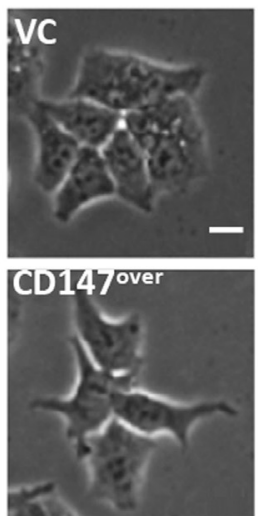

C

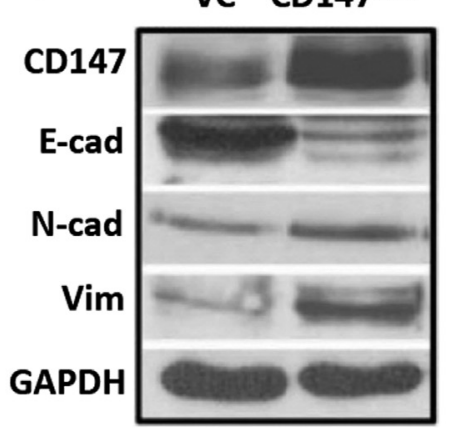

D

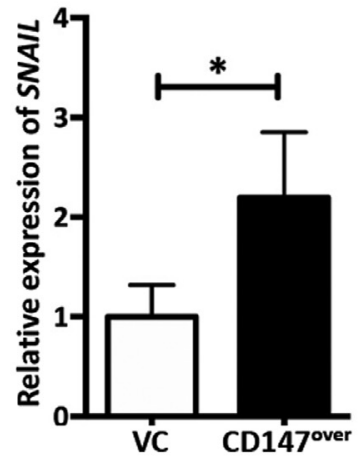

E Blank
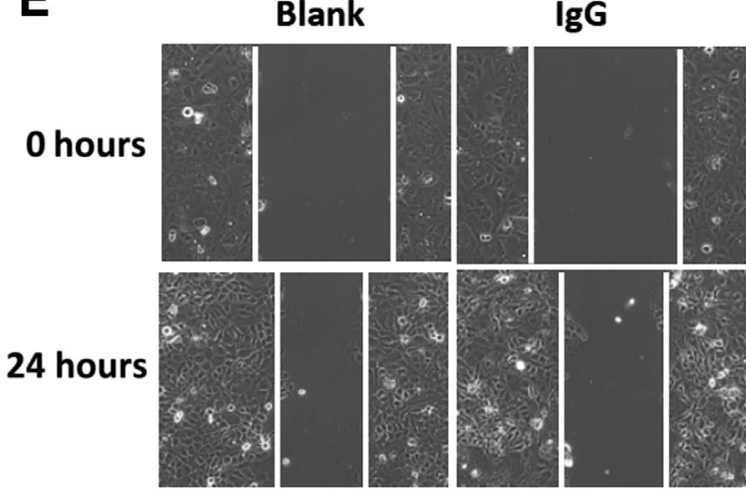

Anti-CD147

F
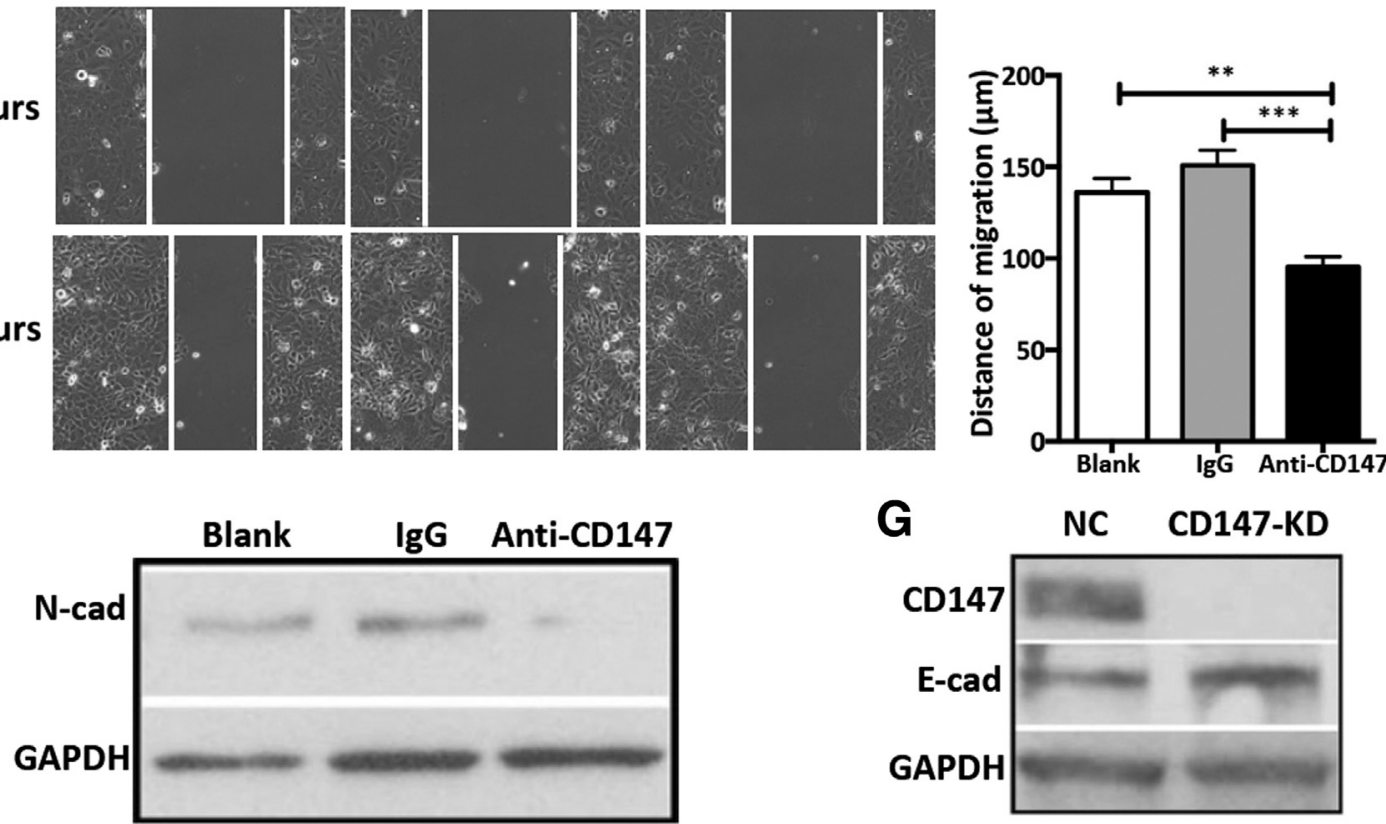

G

NC CD147-KD

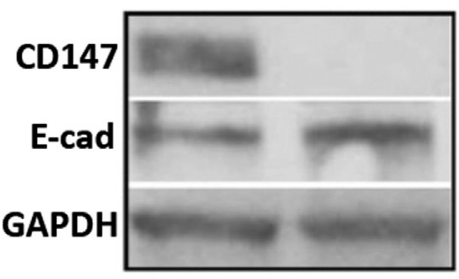

Figure 1 CD147 induces epithelial-to-mesenchymal transition (EMT) in human endometrial cells. A: Representative photographs and statistical analysis of migration of CD147-overexpressing (CD147 ${ }^{\text {over }}$ ) Ishikawa (ISK) cells at 24 hours. Cells transfected with pCDNA 3.0 were used as the vector control (VC). The transfected cells were selected by $400 \mu \mathrm{g} / \mathrm{mL}$ of G418 for 10 days. B: Morphologic features of CD147 ${ }^{\text {over }}$ ISK cells with spindle shape. C: Western blot analysis of CD147, E-cadherin (E-cad), N-cadherin (N-cad), and vimentin (Vim) in CD147 ${ }^{\text {over }}$ ISK cells. D: Real-time quantitative PCR analysis of mRNA levels of SNAIL expression in $\mathrm{CD} 147^{\text {over }}$ ISK cells. The experiments were repeated three times. E: Representative photographs and statistical analysis of the migration of antiCD147-treated ISK cells. Cells were treated with $10 \mu \mathrm{g} / \mathrm{mL}$ of anti-CD147 antibody or normal IgG for 24 hours. F: Western blot analysis of N-cad in antiCD147-treated ISK cells. G: Western blot analysis of CD147 and E-cad in CD147 knockdown (KD) ISK cells. ISK cells transduced with the CD147 shRNA lentivirus and stable cell line were selected by $4 \mu \mathrm{g} / \mathrm{mL}$ of puromycin. Glyceraldehyde-3-phosphate dehydrogenase (GAPDH) was used as the loading control. Data are expressed as means $\pm \mathrm{SEM}(\mathbf{A}, \mathbf{D}$, and $\mathbf{E})$. ${ }^{*} P<0.05,{ }^{* *} P<0.001$, and ${ }^{* * *} P<0.0001$. Scale bar $=10 \mu \mathrm{m}(\mathbf{B})$. Original magnification, $\times 10(\mathbf{A}$ and $\mathbf{E})$. 


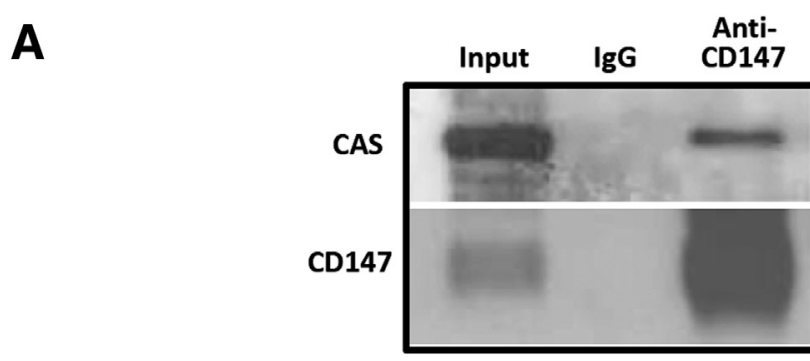

B

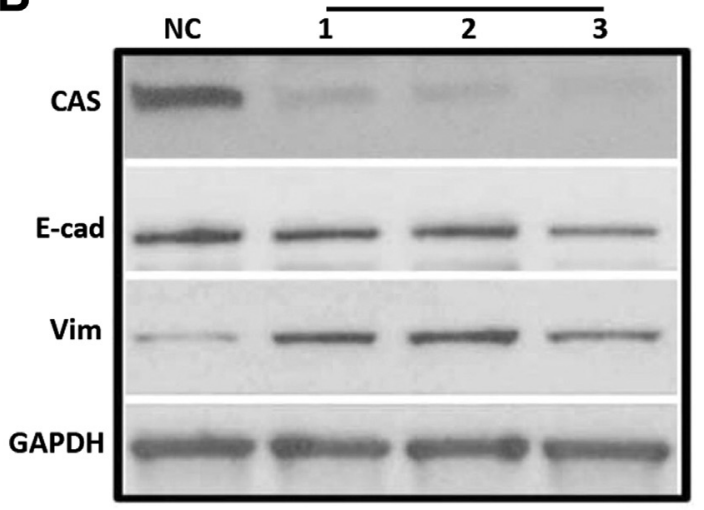

D
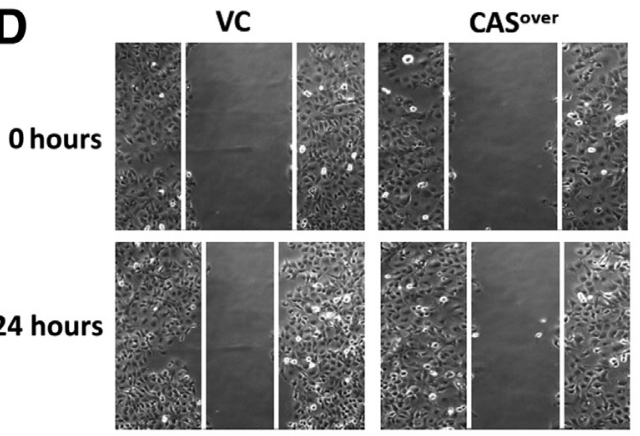

E

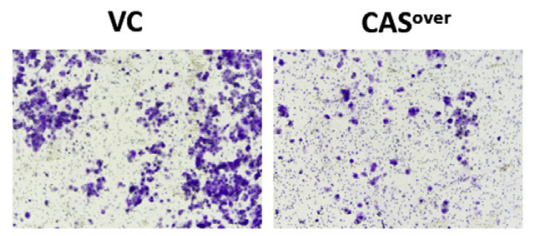

C
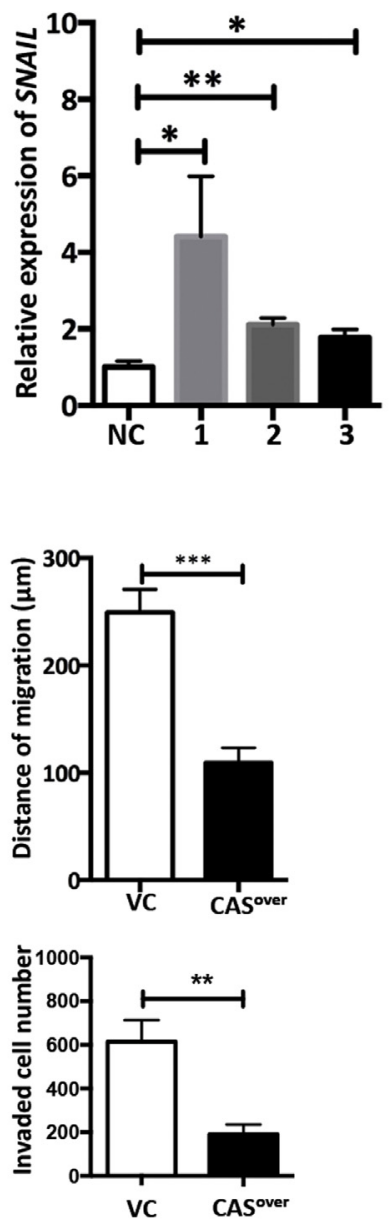

Figure 2 Cellular apoptosis susceptibility protein (CAS) interacts with CD147 and negatively regulates epithelial-to-mesenchymal transition (EMT). A: Western blot analysis of CAS and CD147 in the immunoprecipitation products from Ishikawa (ISK) cells. Immunoprecipitation assay was conducted with anti-CD147 antibody. B: Silencing of CAS-induced EMT in ISK cells. ISK cells were transfected with three differently designed CAS siRNAs at a final concentration of $50 \mathrm{nmol} / \mathrm{L}$, and the total protein was extracted 5 days after transfection. Reduced E-cadherin (E-cad) and increased vimentin (Vim) is observed in CASsilenced ISK cells compared with the cells transfected with negative control (NC) siRNA. C: mRNA levels of SNAIL in CAS-silenced ISK cells were determined by real-time quantitative PCR. The experiments were repeated three times. D and $\mathbf{E}$ : Representative photographs and statistical analysis of migration (D) and invasion (E) of CASoverexpressing (CAS ${ }^{\text {over }}$ ) ISK cells at 24 hours. ISK cells transduced with the CAS ${ }^{\text {over }}$ lentivirus and lentivirus vector control (VC), and stable cell line was selected by $4 \mu \mathrm{g} / \mathrm{mL}$ of puromycin. Data are expressed as means $\pm \mathrm{SEM}(\mathbf{C}-\mathbf{E}) .{ }^{*} P<0.05$, $* * P<0.01$, and $* * * P<0.0001$. Original magnification, $\times 10$ (D and E). GAPDH, glyceraldehyde3-phosphate dehydrogenase. was performed as described previously. ${ }^{24}$ Briefly, cell protein $(30 \mu \mathrm{g}$ per lane) was subjected to SDS-PAGE electrophoresis and was transferred onto nitrocellulose membranes. The transferred membrane was blocked with 5\% skim milk in tris-buffered saline and Tween 20 for 1 hour and was incubated with primary antibodies (Table 1 ) at $4{ }^{\circ} \mathrm{C}$ overnight. Then the membrane was washed three times with trisbuffered saline and Tween 20 and incubated with appropriate peroxidase-conjugated secondary antibodies for 1 hour. The membrane was washed three times with trisbuffered saline and Tween 20 and then detected by enhanced chemiluminescence (GE Healthcare). Glyceraldehyde-3-phosphate dehydrogenase and $\beta$-actin were used as loading control.

\section{Immunofluorescent Staining}

Human samples or coverslips with cells (fixed with $4 \%$ paraformaldehyde in phosphate-buffered saline for 15 minutes) were incubated for 1 hour in blocking solution (5\% normal donkey serum and $1 \%$ bovine serum albumin diluted in phosphate-buffered saline). Diluted primary antibody of rabbit anti- $\beta$-catenin (1:200, Cell Signaling Technology, Danvers, MA) was added on specimen overnight at $4^{\circ} \mathrm{C}$. The primary antibody solutions were removed from the slides, and then the slides were washed three times $(5 \mathrm{mi}-$ nutes each) with $0.05 \%$ Tween 20 in $1 \times$ phosphate-buffered saline. Secondary antibodies (Alexa 488-conjugated donkey-anti-rabbit, 1:500, Invitrogen) were added and 
A

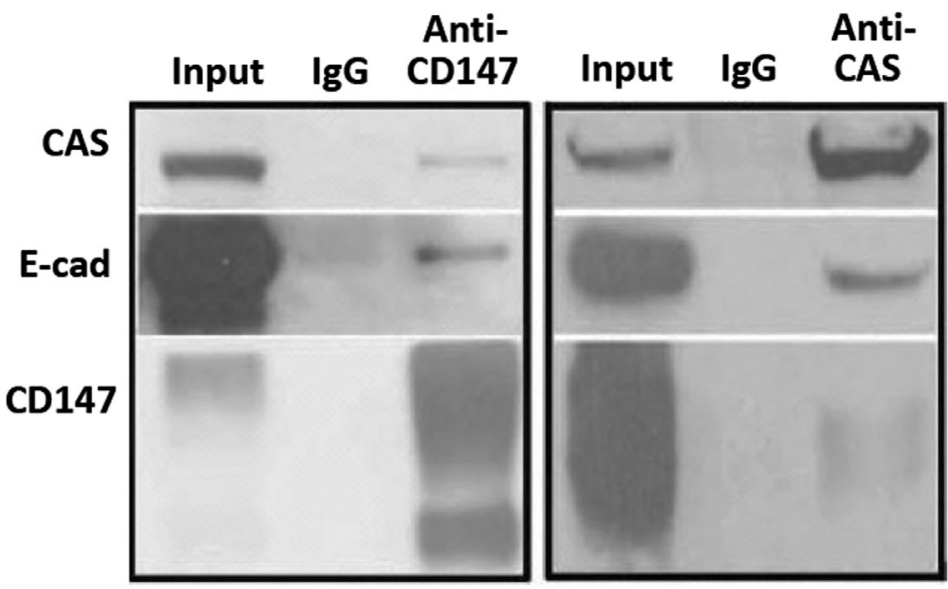

B

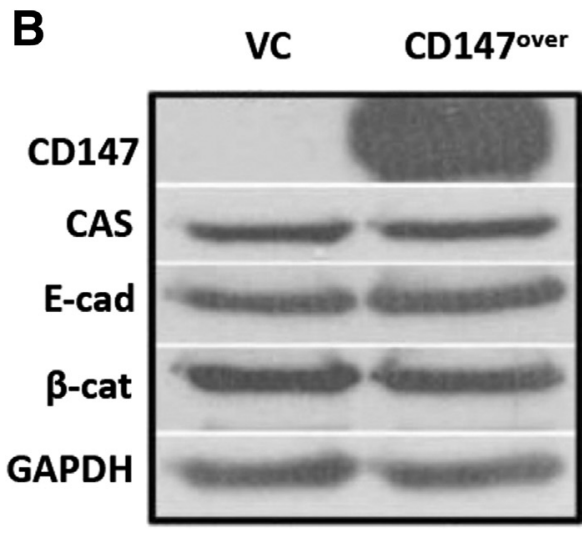

D

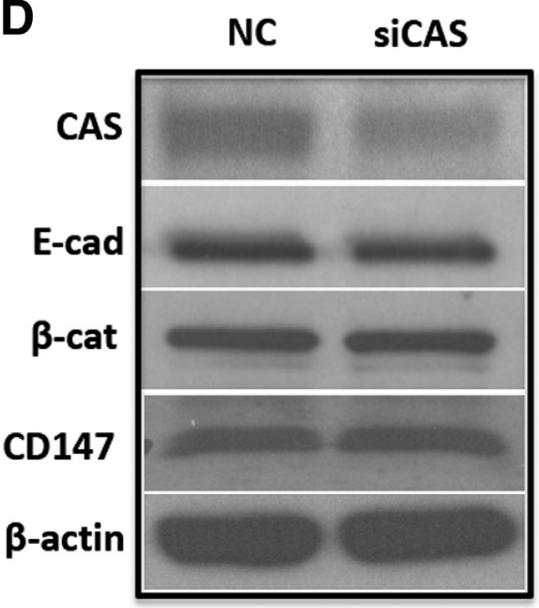

C

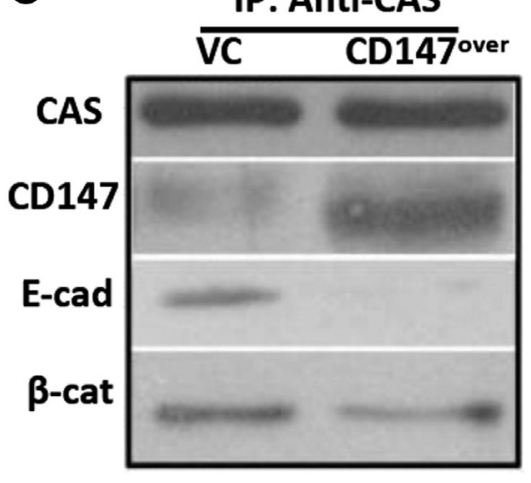

E

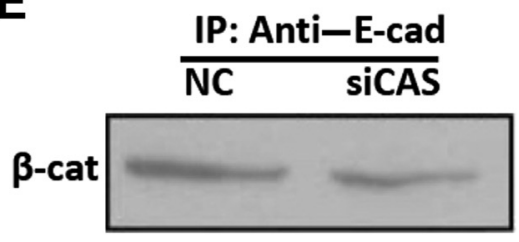

$\mathbf{F}$

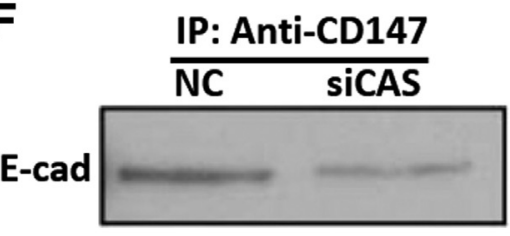

Figure $3 \quad$ CD147 is associated with cellular apoptosis susceptibility protein (CAS)/E-cadherin $(\mathrm{E}$-cad) $/ \beta$-catenin ( $\beta$-cat) complex. A: Western blot analysis of the immunoprecipitation (IP) products from Ishikawa (ISK) cells. IP assay was conducted with anti-CD147 and anti-CAS antibodies, and CD147, CAS, and E-cad were detected by Western blot analysis. B: Input control for C, showing no change in total protein levels of CAS, $\mathrm{E}$-cad, and $\beta$-cat in ISK cells after CD147overexpressing (CD147 ${ }^{\text {over }}$ ) plasmid transfection at 48 hours. CD147 $7^{\text {over }}$ plasmid was introduced into ISK cells, and total cell lysate was collected at 48 hours after transfection. C: Western blot analysis of the IP products from CD147 ${ }^{\text {over }}$ ISK cells at 48 hours after transfection. IP assay was conducted with anti-CAS antibody, and CAS, CD147, E-cad, and $\beta$-cat were analyzed by Western blot. D: Input control for $\mathbf{E}$ and $\mathbf{F}$, showing no change in total protein levels of CD147, E-cad, and $\beta$-cat in CASsilenced ISK cells at 48 hours after small interfering CAS (siCAS) transfection. Cells were transfected with CAS SiRNA1, 2, and 3 or negative control (NC) siRNA and maintained for 48 hours. E: Western blot analysis of $\beta$-cat in the IP products from CAS-silenced ISK cells at 48 hours after transfection. IP assay was conducted with anti-Ecad antibody. F: Western blot analysis of E-cad in the IP products from CAS knockdown ISK cells at 48 hours after transfection. IP assay was conducted with anti-CD147 antibody. ISK cells were transfected with CAS siRNA 1,2, and 3, and total cell lysate was collected at 48 hours after transfection. GAPDH, glyceraldehyde-3-phosphate dehydrogenase; $\mathrm{VC}$, vector control. incubated for 1 hour at room temperature. The slides were mounted with ProLong Gold Antifade Reagent with TOPRO-3 (Invitrogen) and visualized on a Nikon microscope (Nikon Corp, Tokyo, Japan) using $40 \times$ objectives.

\section{Statistical Analysis}

All data were collected by blinded investigators (C.W., J.Z., H.C., and L.L.T.). Statistical significance for comparison between two measurements was determined by $t$-test. One-way analysis of variance was used for evaluation of three measurements. All statistical analyses were performed using Prism software version 6.0 (GraphPad Software, San Diego, CA). Differences were considered statistically significant at $P<0.05$.

\section{Results}

CD147 Promotes Endometrial EMT-Associated Cell Migration

The involvement of CD147 in EMT of endometrium was first examined. CD147 or immunodepleted CD147 was overexpressed in the human endometrial adenocarcinoma cell line ISK and EMT assessed by monitoring cell migration, 
A

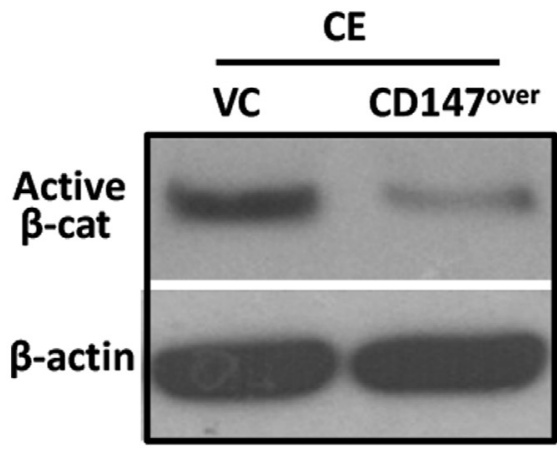

NE

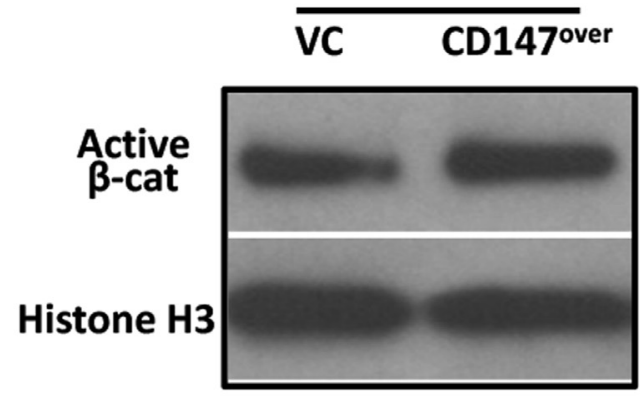

C

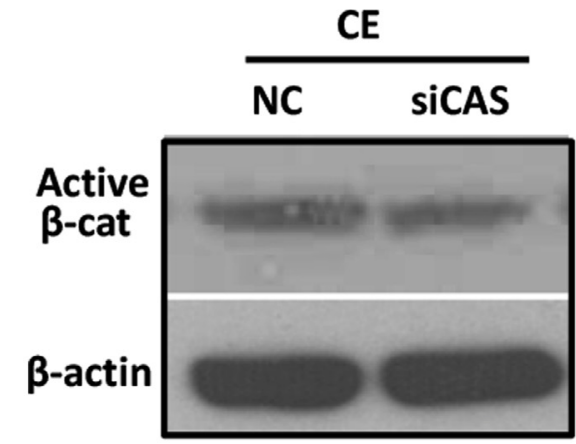

NE

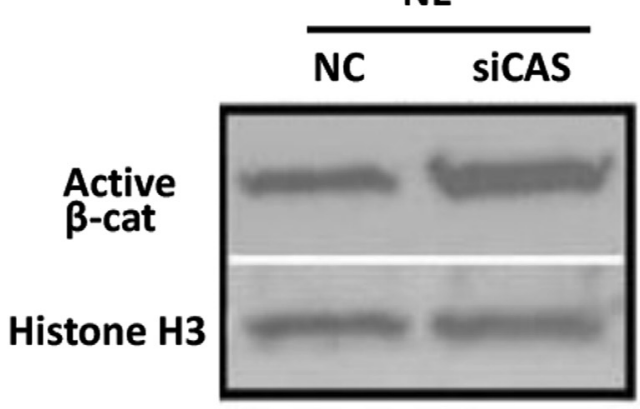

B
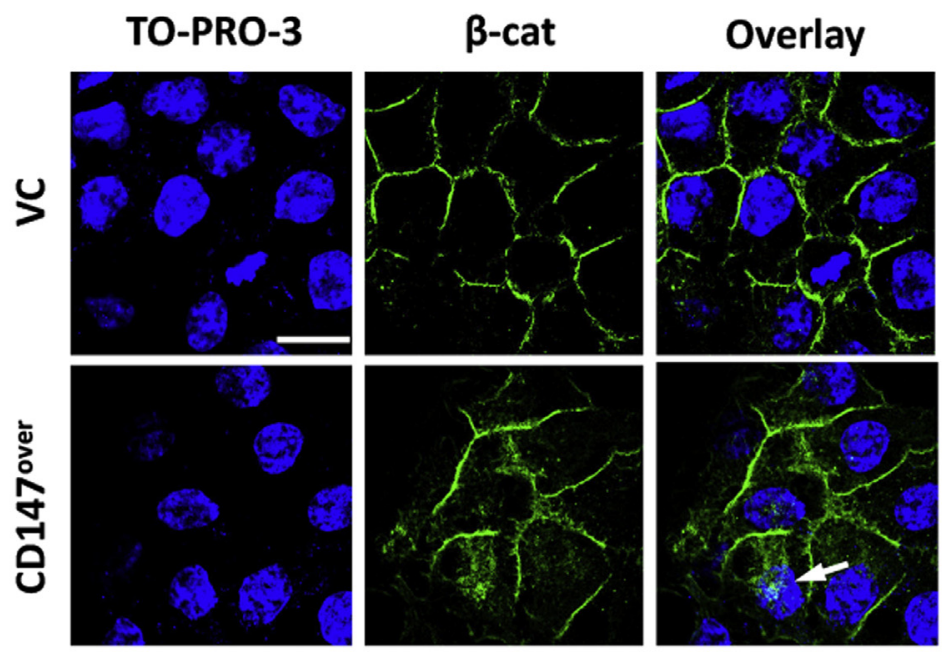

D
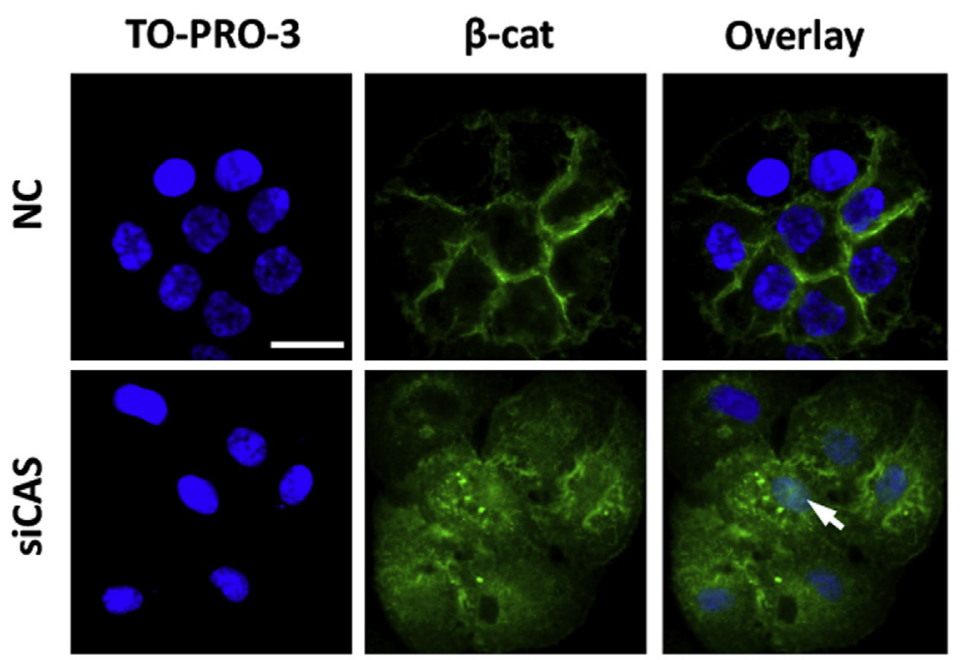

Figure $4 \mathrm{CD} 147$ overexpression (CD147 ${ }^{\text {over }}$ ) and knockdown of cellular apoptosis susceptibility protein (CAS) increases nuclear $\beta$-catenin ( $\beta$-cat). A: Western blot analysis of active $\beta$-cat in the nuclear extracts (NE) and cytoplasmic extracts (CE) of CD147 ${ }^{\text {over }}$ Ishikawa (ISK) cells. Cells transfected with pcDNA 3.0 were used as the vector control (VC). The transfected cells were selected by $400 \mu \mathrm{g} / \mathrm{mL}$ of G 418 for 10 days. B: Immunofluorescent staining of $\beta$-cat in CD147 ${ }^{\text {over }}$ ISK cells. C: Western blot analysis of active $\beta$-cat in the NE and CE of CAS-silenced ISK cells. Cells were transfected with CAS siRNA 1, 2, and 3 or negative control (NC) siRNA and maintained for 3 days. D: Immunofluorescent staining of $\beta$-cat in CAS-silenced ISK cells. $\beta$-actin and histone $\mathrm{H} 3$ were used as loading control. Arrows show the nuclear $\beta$-cat (B and $\mathbf{D}$ ). Nuclei were counterstained with T0-PRO-3 (Thermo Fisher Scientific, Waltham, MA). Scale bar $=20 \mu \mathrm{m}$ (B and D). siCAS, small interfering cellular apoptosis susceptibility protein.

morphologic changes, and EMT markers expression. Overexpression of CD147 dramatically promoted cell migration in ISK cells (Figure 1A). The means \pm SEM migration distance in 24 hours was significantly longer in CD147-overexpressing cells $(211.6 \pm 9.0 \mu \mathrm{m})$ compared with vector control $(127.3 \pm 7.5 \mu \mathrm{m})$. CD147-overexpressing ISK cells transited from cuboidal morphologic structure to a spindle-like, elongated shape compared with the control (Figure 1B). Results of 


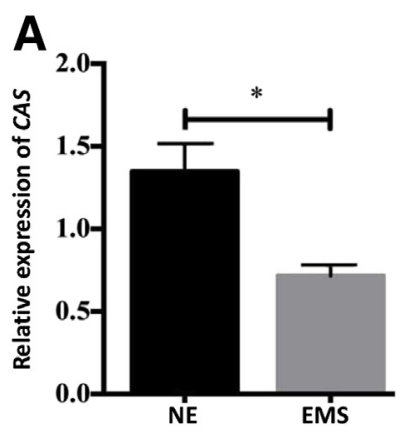

C
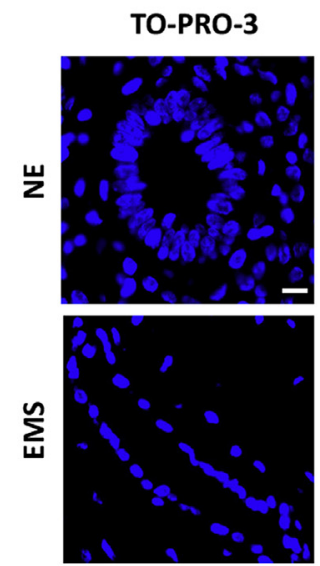

B

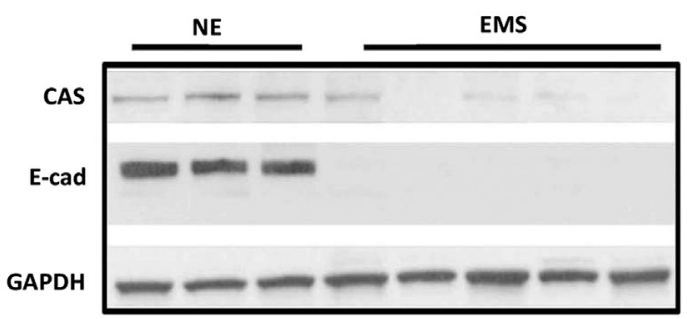

$\beta$-cat
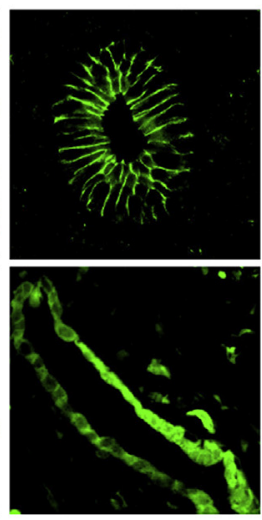

\section{Overlay}
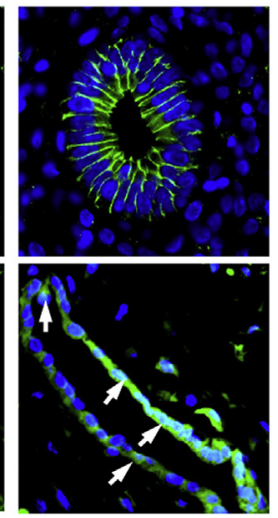

Figure 5 Down-regulation of cellular apoptosis susceptibility protein (CAS) is associated with decreased E-cadherin (E-cad) in human endometriotic lesions. A: mRNA levels of CAS expression in human endometriosis (EMS) tissues were determined by real-time quantitative PCR compared with normal endometrium (NE), The experiments were repeated three times. B: Representative results from Western blot analysis show the protein levels of CAS and E-cad are significantly down-regulated in the ovarian ectopic endometria from women with EMS compared with NE from women without EMS. C: Immunofluorescent staining of $\beta$-catenin ( $\beta$-cat) in NE and EMS. Arrows show the nuclear $\beta$-cat (C). Nuclei were counterstained with TO-PRO-3 (Thermo Fisher Scientific). Data are expressed as means $\pm \operatorname{SEM}(\mathbf{A})$. $n=47$ (A, EMS); $n=12$ (A, NE). ${ }^{*} P<0.001$ versus control. Scale bar $=20 \mu \mathrm{m}$ (C). GAPDH, glyceraldehyde-3-phosphate dehydrogenase.
Western blot analysis showed that CD147 overexpression decreased E-cad and increased the expression of N-cad and vimentin (Figure 1C). The real-time quantitative PCR result demonstrated that the expression of EMT-associated gene SNAIL increased in the CD147-overexpressing ISK cells (Figure 1D). In contrast, blocking CD147 function with a characterized anti-CD147 neutralizing antibody significantly reduced cell migration (Figure $1 \mathrm{E}$ ) and $\mathrm{N}$-cad expression (Figure 1F). Consistently, knockdown of CD147 with lentiviral shRNA in ISK cells up-regulated E-cad (Figure 1G). Together, these results indicate that CD147 promotes EMT-associated migration in endometrial cells.

\section{CD147 Interacts with CAS}

CD147 is required for germ cell migration during both spermatogenesis and EMS. ${ }^{14,17}$ To further investigate the protein-protein interaction networks underlying CD147induced cell migration, CD147-interacting partners were pulled down by co-immunoprecipitation, followed by mass spectrometry identification in spermatogenic GC-2 cells (Supplemental Figure S1 and Supplemental Table S1). From these experiments, CAS was identified as a novel interacting partner of CD147. Interestingly, CD147 also interacts with CAS in ISK cells (Figure 2A).

\section{CAS Negatively Regulates EMT in Endometrium}

Because the role of CAS in EMT has not been explored, the effect of CAS on EMT was determined. CAS was silenced in ISK cells with three different siRNAs, resulting in downregulation of E-cad and up-regulation of vimentin (Figure 2B) as well as increased mRNA expression of EMTpromoting genes SNAIL (Figure 2C). In contrast, overexpression of CAS led to the inhibition of cell migration and invasion (Figure 2, D and E). Taken together, these results indicated that CAS negatively regulates EMT.

\section{CD147 Induces EMT by Disrupting the CAS/E-Cad/ $\beta$-Cat Complex}

During lung tumor metastasis, CD147 induces EMT by activating the $\beta$-cat signaling pathway. ${ }^{20}$ Interestingly, CAS maintains the polarity of human colon epithelial cells by enhancing interaction between E-cad and $\beta$-cat. ${ }^{25}$ Our present finding showed that CD147 and its interacting partner CAS had the opposite effects on EMT. Thus, we speculated that excess CD147 might impair the interaction between CAS and E-cad and lead to the release of $\beta$-cat from E-cad. This in turn facilitates the nuclear translocation of $\beta$-cat and activation of $\beta$-cat signaling pathway. To test this, the interactions between CAS, E-cad, and CD147 were first examined in ISK cells by the co-immunoprecipitation assay using anti-CD147 and antiCAS antibodies, respectively. CAS and E-cad could be pulled down by anti-CD147 antibody, and E-cad and CD147 could be pulled down by anti-CAS antibody, suggesting that the three proteins were associated with each other (Figure 3A). To investigate the interaction dynamics among CD147, CAS, and E-cad, CD147 was overexpressed in ISK 


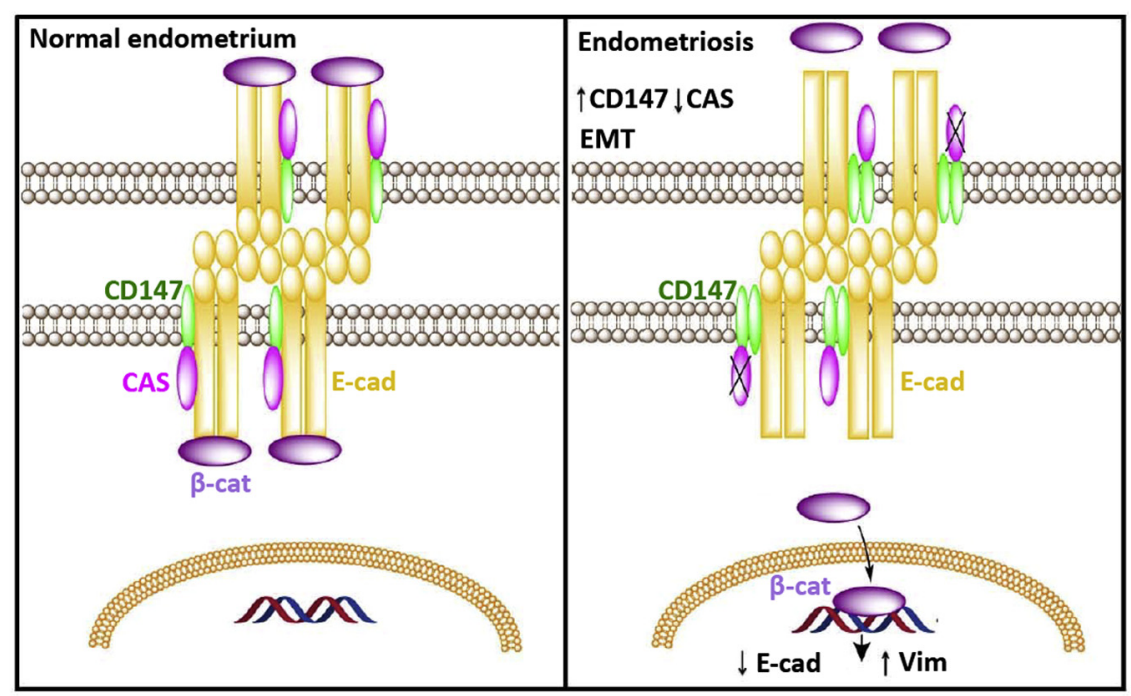

Figure 6 Working model depicting the CD147/ cellular apoptosis susceptibility protein (CAS) regulated epithelial-to-mesenchymal transition (EMT) in endometrial cell. In normal endometrium, CAS binds to $E$-cadherin ( $E$-cad) for enhancing the $E$-cad/ $\beta$ catenin ( $\beta$-cat) complex, preventing the nuclear translocation of $\beta$-cat and maintaining cell-cell junctions. The basal level of CD147 cannot affect the interaction between CAS and E-cad. Excess CD147 impairs the interaction between CAS and E-cad, and down-regulation of CAS further reduces the interaction between $\mathrm{E}$-cad and $\beta$-cat, resulting in release and nuclear translocation of $\beta$-cat. In nucleus, $\beta$-cat can enhance the expression of EMT-associated genes. The endometrial cells undergoing EMT subsequently lose their membrane junctions and transform into motile mesenchymal cells during the progression of endometriosis. The figure was drawn with ChemBioDraw software version 14.0 (PerkinElmer Informatics, Waltham, MA) and Adobe Photoshop CS4 software version 11.0 (Adobe Systems, San Jose, CA). cells, and the total protein was collected at 48 hours after transfection. The total protein expression levels of CAS, Ecad, and $\beta$-cat in the CD147-overexpressing ISK cells were comparable to the vector control (Figure 3B). However, the co-immunoprecipitation results showed that anti-CAS antibody could pull down more CD147, less E-cad, and less $\beta$ cat in the CD147-overexpressing ISK cells compared with the vector control (Figure 3C), suggesting that overexpression of CD147 blocked the interaction between CAS and E-cad, leading to the release of $\beta$-cat from E-cad. To further investigate the interaction among these proteins, CAS was silenced by siRNA, and the total cell protein was collected at 48 hours after transfection. Knockdown of CAS did not affect the total protein levels of E-cad and $\beta$-cat compared with the negative control in 48 hours (Figure 3D) but reduced the interaction between E-cad and $\beta$-cat (Figure 3E). Silencing of CAS also attenuated the interaction between CD147 and E-cad (Figure 3F), suggesting that CD147 was associated with E-cad through CAS. Notably, overexpression of CD147 led to the decreased cytoplasmic $\beta$-cat and increased nuclear $\beta$-cat (Figure 4, A and B). Similarly, knockdown of CAS also caused decreased cytoplasmic $\beta$-cat and increased nuclear $\beta$-cat (Figure 4, $\mathrm{C}$ and D). Taken together, these results suggest that CAS interacts with E-cad to enhance the interaction between E-cad and $\beta$-cat, which can be impaired by excess CD147, resulting in release and nuclear translocation of $\beta$-cat.

\section{Down-Regulation of CAS Is Associated with Decreased $\mathrm{E}-\mathrm{Cad}$ in Human Endometriotic Lesions}

During EMS progression, loss of cell polarity and E-cad of endometrial cells enhanced cell migration and invasion, resulting in the establishment of ectopic lesions. ${ }^{5,6}$ Thus, we speculated that the loss of cell polarity and E-cad in EMS might be associated with the alteration of CAS. To test this, the mRNA levels of CAS were examined in 47 endometriotic lesions and 12 normal endometria from women without EMS by real-time quantitative PCR analysis. The results showed a significant decrease in the mRNA expression of CAS in the endometriotic lesions, with nearly a $50 \%$ decrease compared with the healthy controls (Figure 5A). Consistently, the results of the Western blot analysis showed the decreased protein levels of CAS in the endometriotic lesions in comparison with the healthy controls (Figure 5B). In addition, decreased E-cad was also observed in the endometriotic lesions (Figure 5B) with increased nuclear $\beta$-cat (Figure 5C). These results suggest that down-regulation of CAS promotes EMT of endometriotic cells through $\beta$-cat signaling.

\section{Discussion}

CAS is primarily recognized as a nuclear transport factor; subsequent studies showed that it is also associated with cell polarity. ${ }^{25,26}$ The present study identified CAS as a CD147interacting partner, which negatively regulates EMT by maintaining the stability of the E-cad/ $\beta$-cat complex. The elevated CD147 in EMS promotes EMT by disrupting the $\mathrm{CAS} / \mathrm{E}$-cad/ $\beta$-cat complex, leading to activation of $\beta$-cat pathway (Figure 6). These findings were supported by the analysis of endometriotic samples showing that downregulation of CAS was associated with decreased E-cad and increased nuclear $\beta$-cat.

Overactivation of the $\beta$-cat pathway is associated with the development of EMS.,27-29 Matsuzaki and Darcha ${ }^{4}$ showed the active $\beta$-cat in superficial peritoneal EMS. In addition, the numbers of invasive endometriotic cells were decreased by blocking the transcriptional function of the $\beta$-cat complex in the nucleus, suggesting that abnormal activation of the $\beta$-cat signaling pathway is involved in the invasive phenotype of endometriotic cells. ${ }^{29}$ Taken together with our previous findings of CD147 up-regulation in 
endometriotic samples ${ }^{17}$ both down-regulation of CAS and up-regulation of CD147 contribute to invasive phenotype of endometriotic cells by activation of the $\beta$-cat pathway. Therefore, CD147 and CAS might serve as new biomarkers for early diagnosis of EMS. The alterations of CD147 and CAS in EMS might be caused by different reasons, which need further study.

CD147 is well known to promote cancer metastasis by inducing EMT in colorectal cancer cells, hepatocellular carcinoma cells, and lung carcinoma cells. ${ }^{15,19,20}$ CD147 regulates the migration of endometrial epithelial cells in an MMP-2-independent manner. ${ }^{17}$ In the present study, we demonstrated that CD147 regulates EMT by modulating the interaction dynamics of the CAS/E-cad complex in the endometrium. CD147 is well characterized to stimulate the production of MMPs that digest the extracellular matrix to facilitate cell migration. However, how CD147 regulates the level of MMPs remains largely unknown. To this end, MMPs are also the downstream targets of $\beta$-cat signaling. ${ }^{30}$ Thus, the present findings of the CD147/CAS-regulated $\beta$ cat signaling might reveal an alternative MMP induction mechanism by CD147.

In summary, we propose that this CD147/CAS/E-cad/ $\beta$ cat signaling axis is a critical pathogenic pathway for EMS progression. The present study provides insights into the biological roles of CD147 and CAS in regulating EMT. The capability of CD147 and CAS to regulate $\beta$-cat signaling pathway, as we report here, may have far-reaching implications beyond EMS progression because CD147 and CAS are widely distributed throughout the body and $\beta$-cat signaling is important for many pathologic and physiologic processes, such as tumor metastasis and spermatogenesis.

\section{Acknowledgments}

We thank the individuals who gave informed consent for the use of their samples in this study.

H.C., C.W., and H.C.C. conceived and designed the experiments; C.W., H.C., J.Z., and L.L.T. performed the experiments and analyzed the data; M.Y. and J.L. performed experiments for revised manuscript; F.L. and A.Z.Z. provided intellectual advice; and C.W., H.C., K.L.F., and H.C.C. wrote the paper.

\section{Supplemental Data}

Supplemental material for this article can be found at https://doi.org/10.1016/j.ajpath.2018.03.004.

\section{References}

1. Giudice LC: Clinical practice: endometriosis. N Engl J Med 2010, 362: 2389-2398

2. Goldstein DP, de Cholnoky C, Emans SJ, Leventhal JM: Laparoscopy in the diagnosis and management of pelvic pain in adolescents. J Reprod Med 1980, 24:251-256
3. Eskenazi B, Warner ML: Epidemiology of endometriosis. Obstet Gynecol Clin North Am 1997, 24:235-258

4. Matsuzaki S, Darcha C: Epithelial to mesenchymal transition-like and mesenchymal to epithelial transition-like processes might be involved in the pathogenesis of pelvic endometriosis. Hum Reprod 2012, 27: 712-721

5. Zeitvogel A, Baumann R, Starzinski-Powitz A: Identification of an invasive, $\mathrm{N}$-cadherin-expressing epithelial cell type in endometriosis using a new cell culture model. Am J Pathol 2001, 159:1839-1852

6. Gaetje R, Kotzian S, Herrmann G, Baumann R, Starzinski-Powitz A: Nonmalignant epithelial cells, potentially invasive in human endometriosis, lack the tumor suppressor molecule E-cadherin. Am J Pathol 1997, 150:461-467

7. Heuberger J, Birchmeier W: Interplay of cadherin-mediated cell adhesion and canonical Wnt signaling. Cold Spring Harb Perspect Biol 2010, 2:a002915

8. Kalluri R, Weinberg RA: The basics of epithelial-mesenchymal transition. J Clin Invest 2009, 119:1420-1428

9. Dai C, Stolz DB, Kiss LP, Monga SP, Holzman LB, Liu Y: Wnt/betacatenin signaling promotes podocyte dysfunction and albuminuria. J Am Soc Nephrol 2009, 20:1997-2008

10. Gilles C, Polette M, Mestdagt M, Nawrocki-Raby B, Ruggeri P, Birembaut P, Foidart JM: Transactivation of vimentin by beta-catenin in human breast cancer cells. Cancer Res 2003, 63:2658-2664

11. Ghahhari NM, Babashah S: Interplay between microRNAs and WNT/beta-catenin signalling pathway regulates epithelialmesenchymal transition in cancer. Eur J Cancer 2015, 51:1638-1649

12. Yan L, Zucker S, Toole BP: Roles of the multifunctional glycoprotein, emmprin (basigin; CD147), in tumour progression. Thromb Haemost 2005, 93:199-204

13. Weidle UH, Scheuer W, Eggle D, Klostermann S, Stockinger H: Cancer-related issues of CD147. Cancer Genomics Proteomics 2010, 7:157-169

14. Chen H, Fok KL, Yu S, Jiang JL, Chen ZN, Gui YT, Cai ZM, Chan HC: CD147 is required for matrix metalloproteinases-2 production and germ cell migration during spermatogenesis. Mol Hum Reprod 2011, 17:405-414

15. Wu J, Ru NY, Zhang Y, Li Y, Wei D, Ren Z, Huang XF, Chen ZN, Bian H: HAb18G/CD147 promotes epithelial-mesenchymal transition through TGF-beta signaling and is transcriptionally regulated by Slug. Oncogene 2011, 30:4410-4427

16. Chen H, Lam Fok K, Jiang X, Chan HC: New insights into germ cell migration and survival/apoptosis in spermatogenesis: lessons from CD147. Spermatogenesis 2012, 2:264-272

17. Jin A, Chen H, Wang C, Tsang LL, Jiang X, Cai Z, Chan HC, Zhou X: Elevated expression of CD147 in patients with endometriosis and its role in regulating apoptosis and migration of human endometrial cells. Fertil Steril 2014, 101:1681-1687.e1

18. Wang C, Jin A, Huang W, Tsang LL, Cai Z, Zhou X, Chen H, Chan HC: Up-regulation of Bcl-2 by CD147 through ERK activation results in abnormal Cell survival in human endometriosis. J Clin Endocrinol Metab 2015, 100:E955-E963

19. Xu T, Zhou M, Peng L, Kong S, Miao R, Shi Y, Sheng H, Li L: Upregulation of CD147 promotes cell invasion, epithelial-tomesenchymal transition and activates MAPK/ERK signaling pathway in colorectal cancer. Int J Clin Exp Pathol 2014, 7:7432-7441

20. Sidhu SS, Nawroth R, Retz M, Lemjabbar-Alaoui H, Dasari V, Basbaum C: EMMPRIN regulates the canonical Wnt/beta-catenin signaling pathway, a potential role in accelerating lung tumorigenesis. Oncogene 2010, 29:4145-4156

21. Sun J, Hemler ME: Regulation of MMP-1 and MMP-2 production through CD147/extracellular matrix metalloproteinase inducer interactions. Cancer Res 2001, 61:2276-2281

22. Jiang JL, Zhou Q, Yu MK, Ho LS, Chen ZN, Chan HC: The involvement of HAb18G/CD147 in regulation of store-operated calcium entry and metastasis of human hepatoma cells. J Biol Chem 2001, 276:46870-46877 
23. Huang W, Jin A, Zhang J, Wang C, Tsang LL, Cai Z, Zhou X, Chen H, Chan HC: Upregulation of CFTR in patients with endometriosis and its involvement in NFkappaB-uPAR dependent cell migration. Oncotarget 2017, 8:66951-66959

24. Wang C, Fok KL, Cai Z, Chen H, Chan HC: CD147 regulates extrinsic apoptosis in spermatocytes by modulating NFkappaB signaling pathways. Oncotarget 2017, 8:3132-3143

25. Jiang MC, Liao CF, Tai CC: CAS/CSE 1 stimulates E-cadherindependent cell polarity in HT-29 human colon epithelial cells. Biochem Biophys Res Commun 2002, 294:900-905

26. Kutay U, Bischoff FR, Kostka S, Kraft R, Gorlich D: Export of importin alpha from the nucleus is mediated by a specific nuclear transport factor. Cell 1997, 90:1061-1071
27. Matsuzaki S, Botchorishvili R, Pouly JL, Canis M: Targeting the Wnt/beta-catenin pathway in endometriosis: a potentially effective approach for treatment and prevention. Mol Cell Ther 2014, $2: 36$

28. Gaetje R, Holtrich U, Karn T, Cikrit E, Engels K, Rody A, Kaufmann M: Characterization of WNT7A expression in human endometrium and endometriotic lesions. Fertil Steril 2007, 88:1534-1540

29. Matsuzaki S, Darcha C: In vitro effects of a small-molecule antagonist of the Tcf/ss-catenin complex on endometrial and endometriotic cells of patients with endometriosis. PLoS One 2013, 8:e61690

30. Wu B, Crampton SP, Hughes CC: Wnt signaling induces matrix metalloproteinase expression and regulates $\mathrm{T}$ cell transmigration. Immunity $2007,26: 227-239$ 